\title{
Falsi sinonimi: omesso / omissato
}

\author{
Sergio Lubello
}

PUBBLICATO: 14 APRILE 2020

\section{Quesito:}

Qualche lettore chiede se il termine omissato, invece di omesso, sia corretto.

\section{Falsi sinonimi: omesso / omissato}

S gombriamo subito il campo dall'equivoco: il participio passato di omettere è omesso; il termine in questione, omissato, non è una variante di omesso, cioè non è un participio di omettere, ma è una forma che risale alla stessa origine latina (il verbo omittere) per via diversa. Alla base c'è un latinismo, omissis, espressione e sostantivo maschile invariabile, abbreviazione della formula ceteris rebus omissis 'omesse le altre cose', che, nella riproduzione di un testo e soprattutto di un documento legale o per scopo legale, serve a indicare che vengono omesse parole o nomi propri o intere frasi che per varie ragioni si ritiene di non dover riprodurre (in quanto estranee all'argomento o alla finalità per cui la riproduzione è fatta o in quanto riguardano veri o presunti segreti di stato che non si devono divulgare). L'omissis è molto frequente negli atti notarili nel caso di informazioni non ritenute indispensabili per chi legge oppure per rispetto della privacy; lo stesso succede in atti normativi in cui o per ragioni di brevità o per evidenziare subito la norma di cui si tratta si omettono parti di testo (come articoli di legge); negli atti giudiziari l'omissis serve a proteggere la privacy o, nel caso di indagini aperte e in corso, il segreto istruttorio.

Il latinismo omissis è documentato dal secondo Ottocento (lo registra il dizionario Tommaseo-Bellini) anche se si diffonde più capillarmente a partire nella seconda metà del Novecento grazie alla stampa periodica (nella cronaca riguardante casi giudiziari, inchieste, indagini della magistratura, ecc.); in particolare si veda lo stralcio tratto da "Panorama" (del 27 settembre 1977, p. 40): "Molti loro documenti sono ancora segreti, altri arrivano ai giudici mutilati, con gli ormai famosi 'omissis".

Negli ultimi decenni gli omissis sono diventati nel linguaggio politico-giornalistico sinonimo di segreti che si vogliono lasciare tali ("un documento pieno di omissis") oppure hanno il significato ancora più esteso di 'le parti tralasciate; le cose non dette' ("relazione con molti omissis"; "confessione con vari omissis"): il termine è insomma uscito dall'ambito più tecnico, giuridico-amministrativo, per indicare un tema, un argomento importante che è taciuto o ignorato deliberatamente in uno scritto o in un discorso.

Dal latinismo omissis è stata derivata - sembrerebbe negli anni 'go del 'goo - la forma verbale in -are, omissare, che significa 'coprire con un provvedimento di omissis', il cui participio, omissato, è usato con funzione aggettivale 'coperto di omissis' (nome omissato, informazione omissata, frase omissata ecc.). Questa è la trafila, per cosi dire, etimologica. Ma vediamo qualche dato in più sulle attestazioni e sulle registrazioni lessicografiche.

Documentato per la prima volta in un articolo della "Stampa" del 23 aprile I994 (p. II, Interno), omissare (specie nella forma omissato) costituisce un tecnicismo che attiene a settori precisi, giudiziario e amministrativo, come si ricava dalle varie attestazioni giornalistiche fornite dai repertori e recuperabili attraverso la ricerca in Google libri; ne riportiamo solo alcune più significative che 
chiariscono l'ambito e il contesto d'uso (si veda in particolare la seconda attestazione, dal "Corriere della sera", in cui Vittorio Grevi definisce il termine omissato un "terribile neologismo").

[...] nel corso degli interrogatori sono stati sottoposti agli indagati i contenuti di numerose intercettazioni. In una è stato omissato il nome di Franco Coppi, che comunque risulta "persona estranea alle indagini". ("Corriere della sera", $17 / 3 / 1996$, p. 4)

[...] qualora il magistrato procedente ritenga di non utilizzare simili conversazioni, si è diffusa la prassi (suggerita talora anche in sede parlamentare) di non indicare negli atti resi noti alle parti il nome del membro del Parlamento, e di coprire con "omissis" il contenuto delle sue conversazioni così intercettate, in modo da renderne impossibile la conoscenza. E ciò è avvenuto, per quanto risulta, anche nell'inchiesta milanese su Antonveneta, dove sono state "omissate" (per usare questo terribile neologismo) tutte le conversazioni di membri del Parlamento casualmente intercettate su utenze di terze persone. ("Corriere della sera", i4 $/ 8 / 2005$, p. 32, Cultura)

[...] tra gli atti depositati e consegnati su dvd ai legali, l'elenco delle telefonate (data, ora, chiamante, chiamato) è stato omissato, e solo negli uffici dei pm gli avvocati hanno potuto visionare l'originale, facendosi almeno un'idea dei nomi dei parlamentari in questione. ("Corriere della sera", 20/3/2007, p. 24, Cronache)

[Antonio] Catricalà ha spiegato che Unicredit ha scelto di adottare "regole generali di astensione per evitare conflitti di ruolo e Chinese Walls (muraglie cinesi) per evitare scambi di informazione". Le misure prevedono che i consiglieri in conflitto di interesse ricevano un ordine del giorno del Cda "omissato" per quanto riguarda i punti relativi a investment banking e assicurazioni. I consiglieri dovranno inoltre lasciare la riunione quando i punti saranno discussi. ("Il Piccolo", I4/2/2008, p. 6, Economia \& Porto)

Il Viminale [...] gli ha negato il programma di protezione per aver parlato della trattativa e degli interlocutori politici di Cosa nostra oltre il termine previsto dalla legge, e [Gaspare] Spatuzza si difende: "Quando ho deciso di collaborare sapevo che con le mie dichiarazioni avrei fatto riaprire processi già chiusi, e quindi sapevo di entrare in conflitto con la magistratura, con la polizia, con in servizi segreti e con la politica. Ero da solo, ho avuto paura e ho deciso di omissare alcune questioni. Giuridicamente ho sbagliato ed è giusto che venga punito per questo, ma moralmente ritengo di aver fatto la scelta giusta". ("Corriere della sera", 4/2/20II, p. 20, Cronache)

Diviene così opportuno invitare i magistrati a scelte oculate nel redigere i provvedimenti giudiziari: "Si attengano a onere di sobrietà contenutistica, eventualmente valutando se omissare (così nel documento, ndr), nelle conversazioni comunque rilevanti, i riferimenti a cose o persone, se non strettamente necessari". ("Avvenire", 30/7/2016, p. 9, Attualità).

Il termine, dunque, è non solo recente ma anche raro e di ristretto uso scritto, tanto che è tuttora assente da molti dizionari monovolume: è invece registrato (e datato i996) nella seconda edizione del GRADIT (del 2007, che non registra, invece, omissare), nel repertorio dei Neologismi Treccani (s.v. omissare e omissato), nell'Osservatorio dei neologismi italiani, ONLI, e nel Supplemento 2009 del GDLI.

\section{Cita come:}

Sergio Lubello, Falsi sinonimi: omesso /omissato " "Italiano digitale", 2019, XIII, 2020/2 (aprilegiugno)

DOI: $10.35948 / 2532-9006 / 2020.3305$ 